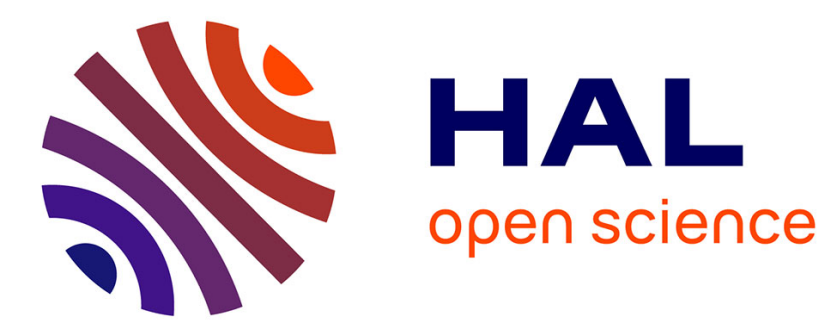

\title{
Modeling and Control of a Redundant Tensegrity-based Manipulator
}

\author{
Jeremy Begey, Marc Vedrines, Pierre Renaud, Nicolas Andreff
}

\section{To cite this version:}

Jeremy Begey, Marc Vedrines, Pierre Renaud, Nicolas Andreff. Modeling and Control of a Redundant Tensegrity-based Manipulator. International symposium on Advances in Robot Kinematics, Jun 2021, Ljubljana, Slovenia. hal-03549321

\section{HAL Id: hal-03549321 \\ https://hal.science/hal-03549321}

Submitted on 31 Jan 2022

HAL is a multi-disciplinary open access archive for the deposit and dissemination of scientific research documents, whether they are published or not. The documents may come from teaching and research institutions in France or abroad, or from public or private research centers.
L'archive ouverte pluridisciplinaire HAL, est destinée au dépôt et à la diffusion de documents scientifiques de niveau recherche, publiés ou non, émanant des établissements d'enseignement et de recherche français ou étrangers, des laboratoires publics ou privés. 


\title{
Modeling and Control of a Redundant Tensegrity-based Manipulator
}

\author{
Jérémy Begey, Marc Vedrines, Pierre Renaud, and Nicolas Andreff
}

\begin{abstract}
Tensegrity-based mechanisms draw attention in particular for their deployability and compliance. However, task-based design and control of such systems are still open topics. In a previous work, a tensegrity-based manipulator was designed to respect the remote center of motion constraint encountered in medical applications. Only the workspace was then analyzed. Here, we develop the kinematic model of this manipulator and then exploit it for the control. Such a manipulator is redundant. The use of redundancy is discussed and evaluated in simulation with two control schemes using Jacobian based controllers.
\end{abstract}

Key words: Tensegrity Mechanisms, Redundant Manipulator, Kinematic Control.

\section{Introduction}

Tensegrities are pre-stressed structures composed of bars and cables, firstly exploited [10] for their high resistance. Several authors focused on actuating these structures to design tensegrity mechanisms. If some structural elements are chosen elastic, these mechanisms can be compliant with controllable stiffness. Therefore, these mechanisms are of high interest when interactions with the environment can occur, particularly when contact management is critical. For instance, it was used for grasping tasks [12], design of flexible mobile robot [7] or to carry out medical procedures [4]. In [4], a tensegrity-based manipulator has been proposed to manage a Remote Center of Motion (RCM) constraint often met in medical procedures. It is introduced in Fig. 1, and presented in detail in section 2. The device is built for quasi-static positioning. Only preliminary analysis of the robot workspace was

Jérémy Begey (jeremy.begey@etu.unistra.fr), ICube-Femto-ST, France

Marc Vedrines, Pierre Renaud, ICube, France

Nicolas Andreff, Femto-ST - France 


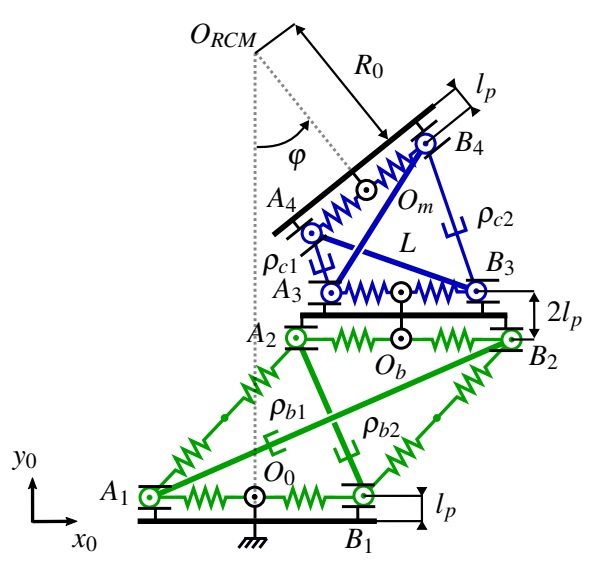

Fig. 1 The considered tensegrity-based manipulator composed of two tensegrity mechanisms: a bar-actuated one in green and a cable-actuated one in blue.

yet achieved. Further development is hampered by the absence of analytical Forward Kinematic (FK) model and the control strategy. In this paper, kinematics are developed and used to investigate manipulator control.

Solutions to the FK problem correspond to stable configurations of the prestressed mechanism. Amongst others, energetic approaches have been proposed [6]. Depending on the type of elastic elements, solving the FK problem remains complex [3,13] and it is often treated numerically [3-5]. In this paper, we show using an energetic approach that an analytical solution can be determined for the considered manipulator. This constitutes the first contribution of the paper.

The proposed manipulator is considered for providing a RCM in the plane. Therefore, this four actuator mechanism is redundant with respect to this task. Control of redundant tensegrities has been considered to achieve shape control while optimizing a given criterion and get for instance time-optimal or energy-optimal control $[1,11]$. In conventional robotics, control of redundant systems is making use of the kinematic Jacobian [9]. We show in this paper that the Jacobian matrix can be derived analytically so such methods are well suited. Control schemes exploiting the redundancy are then proposed and evaluated in simulation as a second contribution.

The manipulator is described and modeled in Section 2. The control schemes and the obtained results in simulation are then discussed in Section 3. Conclusions are finally drawn in Section 4.

\section{Description and modeling of the manipulator}

The considered mechanism is planar and composed of two serially assembled Xshaped tensegrities called Snelson Crosses (SC) [10]. A bar-actuated SC, displayed at the bottom of Fig. 1, is mounted on the base. It is actuated by modifying the 
lengths $\rho_{b 1}$ and $\rho_{b 2}$ of the bars attached to nodes $A_{1}, B_{2}$ and $B_{1}, A_{2}$ respectively. The SC on top is cable-actuated with control of the lengths $\rho_{c 1}$ and $\rho_{c 2}$ of nonelastic cables attached to nodes $A_{3}, A_{4}$ and $B_{3}, B_{4}$ respectively. The bars of this SC have a length $L$. Following [4], analysis is conducted while neglecting gravity forces. Thanks to the intermediate component placed between the SCs, the static models of the SCs are decoupled, and the manipulator can be assessed by independently analyzing each SC. For sake of symmetry, two elementary springs are placed on the top and bottom sides of each SC. Two serially connected springs are then similarly placed between the nodes $A_{1}, A_{2}$ and $B_{1}, B_{2}$, with all springs of stiffness $2 K$ and free-length $l_{0 c} / 2$ (resp. $l_{0 b} / 2$ ) for the cable- (resp. bar-) actuated SC. We will refer in the following to a spring as the equivalent elastic element of the two serially attached springs on each side. The end-effector of the manipulator is the platform on top of the mechanism (Fig. 1). For given position of the remote center of motion $O_{R C M}$ and orientation $\varphi$, it is simple to determine the corresponding coordinates of $O_{m}$. The FK problem consists then to determine from the joint coordinates $\mathbf{q}=\left[\rho_{b 1}, \rho_{b 2}, \rho_{c 1}, \rho_{c 2}\right]^{T}$ the end-effector pose $\mathbf{x}=[x, y, \varphi]^{T}$, with $(x, y)$ the position of $O_{m}$ the barycentre of points $A_{4}$ and $B_{4}$, and $\varphi$ the orientation.

In [4], it was observed with numerical analysis that the bar-actuated SC shape is a parallelogram for all reachable configurations. We could also observe that these configurations seem diamond-shaped. Thus, all springs would be of same length which would be expressed in Eq. (1), with $L_{A_{i} B_{j}}$ the length between nodes $A_{i}$ and $B_{j}$. In [2], the stable configuration of a cable-actuated SC was analyzed and Eq. (2) was in addition demonstrated for zero free-length springs. Again using numerical analysis, Eq. (2) was observed to be valid in [4] for springs with non-zero free-length.

$$
L_{A_{1} B_{1}}=\frac{1}{2} \sqrt{\rho_{b 1}^{2}+\rho_{b 2}^{2}} \quad \text { (1) } \quad L_{A_{3} B_{3}}=\sqrt{L^{2}-\rho_{c 1} \rho_{c 2}}
$$

To verify the validity of expressions (1) and (2) for springs with non-zero freelength, the conditions of tensegrity mechanism equilibrium configuration [5] are derived analytically. Let $\mathbf{q}_{1}=\left[\rho_{b 1}, \rho_{b 2}\right]^{T}$ and $\mathbf{q}_{2}=\left[\rho_{c 1}, \rho_{c 2}\right]^{T}$ be the sets of joint coordinates of the bar- and the cable-actuated SCs. The SCs are compliant so $\mathbf{q}_{u i}$ designates sets of unconstrained joint coordinates. Existence of a stable static equilibrium can then be expressed from the mechanism potential energy $U_{i}\left(\mathbf{q}_{i}, \mathbf{q}_{u i}\right)$ (Eq. (3)), verifying also that the tensions in the elements in traction are positive.

$$
\frac{\partial U_{i}\left(\mathbf{q}_{i}, \mathbf{q}_{u i}\right)}{\partial \mathbf{q}_{u i}}=0 \quad \frac{\partial^{2} U_{i}\left(\mathbf{q}_{i}, \mathbf{q}_{u i}\right)}{\partial \mathbf{q}_{u i}^{2}}>0 \quad i=\{1,2\}
$$

For both SC, the potential energy is written with $\mathbf{q}_{u 1}=\left[L_{A_{1} B_{1}}, L_{A_{1} A_{2}}, L_{B_{1} B_{2}}\right]^{T}$ for the bar-actuated SC and $q_{u 2}=L_{A_{3} B_{3}}$ for the cable-actuated SC, having respectively three and one unconstrained coordinates [3]. It gives:

$$
\begin{aligned}
U_{1} & =\frac{1}{2} K\left(\left(L_{A_{2} B_{2}}\left(\mathbf{q}_{1}, \mathbf{q}_{u 1}\right)-l_{0 b}\right)^{2}+\sum_{j=1}^{j=3}\left(\mathbf{q}_{u 1}(j)-l_{0 b}\right)^{2}\right) \\
U_{2} & =\frac{1}{2} K\left(\left(L_{A_{4} B_{4}}\left(\mathbf{q}_{2}, q_{u 2}\right)-l_{0 c}\right)^{2}+\left(q_{u 2}-l_{0 c}\right)^{2}\right)
\end{aligned}
$$


The first and second derivatives are then derived and the lengths in $\mathbf{q}_{u i}$ are replaced by their proposed expressions (1) or (2). It then leads that all the first derivatives are equal to zero. The expressions of the second derivatives are not detailed here for paper clarity, but are strictly positive if the components of $\mathbf{q}_{i}$, the elongation and the stiffness of the springs are strictly positive, which is always verified. Therefore, these configurations correspond to tensegrity configurations. However, here the springs of the bar- (resp. cable-) actuated SC present a free-length $l_{0 b}$ (resp. $l_{0 c}$ ) and a maximal elongation $\delta_{e b}\left(\right.$ resp. $\delta_{e c}$ ). To ensure admissible tensions for springs and cables in a practical implementation, the lengths of the springs must be included in $\left[l_{0 b}, l_{0 b}+\delta_{e b}\right]$ (resp. $\left.\left[l_{0 c}, l_{0 c}+\delta_{e c}\right]\right)$ for the bar- (resp. cable-) actuated SC.

Stability analyses showed that the configuration of the mechanism is independent of the spring stiffness and free-length (Eq. (1) and (2)). Modeling of the manipulator is then reduced to the manipulation of geometrical relationships and the forward static model can be derived analytically. The pose of the end-effector is expressed as:

$$
\begin{array}{rlrl}
\mathbf{x} & =\left[\begin{array}{l}
O_{m} \\
\varphi_{m}
\end{array}\right] \quad \text { with } & O_{m}=O_{b}+\left[\begin{array}{c}
0 \\
2 l_{p}
\end{array}\right]+\left[\begin{array}{l}
x_{c} \\
y_{c}
\end{array}\right], \quad O_{b}=O_{0}+\left[\begin{array}{l}
x_{b} \\
y_{b}
\end{array}\right] \\
\left\{\begin{array}{l}
x_{b}=\frac{\rho_{b 1}^{2}-\rho_{b 2}^{2}}{4 L_{A_{1} B_{1}}} \\
y_{b}=\frac{\rho_{b 1} \rho_{b 2}}{2 L_{A_{1} B_{1}}}
\end{array}\right. & \left\{\begin{array}{l}
x_{c}=\frac{\rho_{c 1}^{2}-\rho_{c 2}^{2}}{4 L_{A_{3} B_{3}}} \\
y_{c}=\left(\frac{\rho_{c 1}+\rho_{c 2}}{2}\right) \sqrt{1+\frac{\left(\rho_{c 1} \rho_{c 2}-\rho_{c 1}^{2}\right)^{2}}{4 \rho_{c 1}^{2}\left(\rho_{c 1} \rho_{c 2}-L^{2}\right)}}
\end{array}\right.
\end{array}
$$

and

$$
\varphi_{m}=2 \arccos \left(\frac{\rho_{c 1}-\rho_{c 2}}{2 L_{A_{3} B_{3}}}\right)-\pi
$$

The kinematic Jacobian matrix $\mathbf{J}$ of the manipulator maps the Cartesian velocities of the end-effector $\dot{\mathbf{x}}$ with respect to the joint velocities $\dot{\mathbf{q}}: \dot{\mathbf{x}}=\mathbf{J} \dot{\mathbf{q}}$. The pose of the endeffector is defined by three coordinates while four actuators are used. The system is then redundant and $\mathbf{J}$ is a $3 \times 4$ matrix. The kinematic Jacobian matrices $\mathbf{J}_{b}$ and $\mathbf{J}_{c}$ of the bar- and cable-actuated SC respectively are given in (8) and (9).

$$
\begin{gathered}
\mathbf{J}_{b}=\left[\frac{\partial \mathbf{x}}{\partial \mathbf{q}_{1}}\right]=\left[\begin{array}{c}
\frac{\partial O_{m}}{\partial \mathbf{q}_{1}} \\
0
\end{array}\right]=\frac{1}{8 L_{A_{1} B_{1}}^{3}}\left[\begin{array}{cc}
\frac{\rho_{b 1}\left(\rho_{b 1}^{2}+3 \rho_{b 2}^{2}\right)}{2} & -\frac{\rho_{b 2}\left(3 \rho_{b 1}^{2}+\rho_{b 2}^{2}\right)}{2} \\
\rho_{b 2}^{3} & \rho_{b 1}^{3} \\
0 & 0
\end{array}\right] \\
\mathbf{J}_{c}=\left[\frac{\partial \mathbf{x}}{\partial \mathbf{q}_{2}}\right]=\left[\begin{array}{l}
\frac{\partial O_{m}}{\partial \mathbf{q}_{2}} \\
\frac{\partial \varphi_{m}}{\partial \mathbf{q}_{2}}
\end{array}\right]=\frac{1}{8 L_{A_{3} B_{3}}^{3}}\left[\begin{array}{cc}
E_{1}\left(\rho_{c 1}, \rho_{c 2}\right) & -E_{1}\left(\rho_{c 2}, \rho_{c 1}\right) \\
\frac{E_{2}\left(\rho_{c 1}, \rho_{c 2}\right)}{\sqrt{4 L^{2}-\left(\rho_{c 1}+\rho_{c 2}\right)^{2}}} & \frac{E_{2}\left(\rho_{c 2}, \rho_{c 1}\right)}{\sqrt{4 L^{2}-\left(\rho_{c 1}+\rho_{c 2}\right)^{2}}} \\
-E_{3}\left(\rho_{c 1}, \rho_{c 2}\right) & E_{3}\left(\rho_{c 2}, \rho_{c 1}\right)
\end{array}\right]
\end{gathered}
$$


with

$$
\begin{aligned}
& E_{1}\left(\rho_{i}, \rho_{j}\right)=4 L^{2} \rho_{i}-3 \rho_{j} \rho_{i}^{2}-\rho_{j}^{3} \\
& E_{2}\left(\rho_{i}, \rho_{j}\right)=8 L^{4}-4 \rho_{i} L^{2}\left(\rho_{i}+3 \rho_{j}\right)+\rho_{j}\left(3 \rho_{i}-\rho_{j}\right)\left(\rho_{i}+\rho_{j}\right)^{2} \\
& E_{3}\left(\rho_{i}, \rho_{j}\right)=8 L_{A_{3} B_{3}}^{2}\left(1-\rho_{i}\left(\rho_{i}-\rho_{j}\right) / 2 L_{A_{3} B_{3}}^{2}\right)\left(\sqrt{1-\left(\rho_{i}-\rho_{j}\right)^{2} / 4 L_{A_{3} B_{3}}^{2}}\right)^{-1}
\end{aligned}
$$

which leads to

$$
\dot{\mathbf{x}}=\mathbf{J}_{b}\left[\begin{array}{l}
\dot{\rho_{b 1}} \\
\dot{\rho_{b 2}}
\end{array}\right]+\mathbf{J}_{c}\left[\begin{array}{l}
\dot{\rho_{c 1}} \\
\dot{\rho_{c 2}}
\end{array}\right]
$$

and the kinematic Jacobian matrix is finally expressed as

$$
\mathbf{J}=\left[\mathbf{J}_{b}, \mathbf{J}_{c}\right]
$$

\section{Control loop and simulation}

Thanks to the derivation of the kinematic Jacobian of the manipulator in (12), Jacobian based control for redundancy management can be exploited. To carry out a path tracking task in the Cartesian space with a redundant manipulator, the joint velocities are computed as:

$$
\dot{\mathbf{q}}=\mathbf{P} \mathbf{J}^{\dagger}(\mathbf{q})\left(\mathbf{x}-\mathbf{x}^{*}\right)
$$

with $\mathbf{J}^{\dagger}$ the Moore-Penrose pseudo-inverse Jacobian and $\mathbf{P}$ a negative diagonal matrix. Using Eq. (13), the redundancy is not exploited and the pseudo-inverse then minimizes the Euclidean norm of joint velocities. To add secondary tasks, the gradient projection method [9] can be used to take advantage of the null-space of $\mathbf{J}$ and optimize a given cost function $h(\mathbf{q})$. The joint velocities are then expressed as:

$$
\dot{\mathbf{q}}=\mathbf{P}\left(\mathbf{J}^{\dagger}(\mathbf{q})\left(\mathbf{x}-\mathbf{x}^{*}\right)+k\left[\mathbf{I}-\mathbf{J}^{\dagger}(\mathbf{q}) \mathbf{J}(\mathbf{q})\right]\left(\frac{\partial h(\mathbf{q})}{\partial \mathbf{q}}\right)^{T}\right)
$$

with $k$ a positive scalar. With the control scheme presented in (13), the trajectory is controlled in the Cartesian space but not in the joint space. However, if the actuator limit positions are reached, trajectory distortions will be induced. The RCM constraint can thus be violated, which is not acceptable (especially in a medical context). Also, the control of a tensegrity mechanism requires that the cables and springs are in tension at all time. To respect this constraint, actuator strokes are chosen accordingly. Moreover, as shown in Eq. (1) and Eq. (2), the spring lengths are directly linked to the joint positions. Therefore, maximizing the distance between the joint positions and their limits will also maximize the distance between the spring length and the lengths at rest and at maximal elongation. This approach is then also interesting to avoid situations with low or high tensions in the springs and cables. As a consequence, a cost function $h(\mathbf{q})$ is proposed to minimize the distance between 


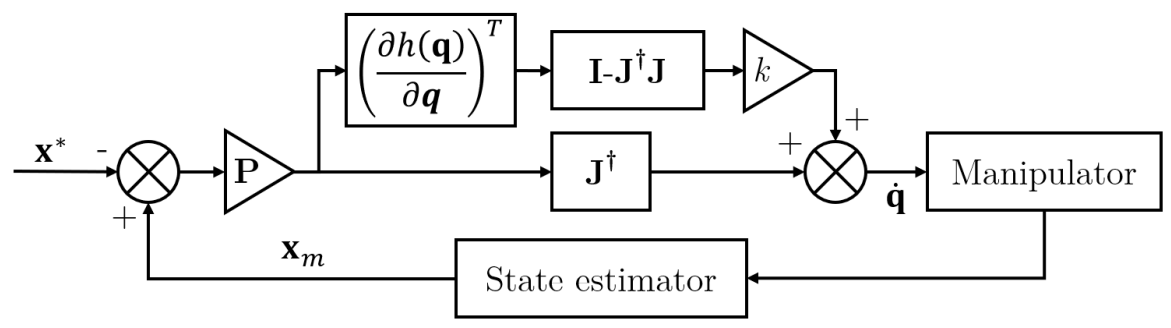

Fig. 2 Proposed control loop.

the measured joint positions and the mean value of the reachable positions following [8]. With $\rho_{b \min }, \rho_{b \max }$ and $\rho_{c \min }, \rho_{c \max }$ the actuator limits of the bar-actuated and the cable-actuated SC respectively, the cost function is written as

$$
\begin{array}{r}
h_{a}=\frac{1}{4} \sum_{i=1}^{2} \underbrace{p_{1}\left(\frac{\rho_{b i}-\alpha_{b i}}{\rho_{b \max }-\alpha_{b i}}\right)^{2}}_{\text {Actuators of the bar-actuated SC }}+\underbrace{p_{2}\left(\frac{\rho_{c i}-\alpha_{c i}}{\rho_{c m a x}-\alpha_{c i}}\right)^{2}}_{\text {Actuators of the cable-actuated SC }} \\
\alpha_{b i}=\left(\rho_{b \max }+\rho_{b \min }\right) / 2 \quad \alpha_{c i}=\left(\rho_{\text {cmax }}+\rho_{c \min }\right) / 2
\end{array}
$$

with $p_{1}$ and $p_{2}$ some coefficients to be tuned.

The obtained control loop is displayed in Fig. 2. A pose feedback $\mathbf{x}_{m}$ is considered. It can be implemented by measuring the joint coordinates and using the forward kinematic model or using an exteroceptive measurement. This choice is not discussed in this paper. We also consider that the motions are slow enough to avoid actuator velocity saturation and the transfer function of the actuators is considered to be unitary.

In order to evaluate the proposed control schemes, dynamic simulation is implemented with the Simscape ${ }^{\mathrm{TM}}$ Toolbox of Matlab Software (MathWorks, USA). This simulation is thereby independent from the models used for control. Weightless springs and cables are considered while the bars have a mass $m_{b}$, the end-effector and the decoupling element a mass $m_{p}$. Parameters are reported in Table 1. In practical implementation there are inevitably dissipative forces. We here simulate the latter with dampers placed in parallel to the springs and in the prismatic joints with a damping coefficient of $20 \mathrm{~N} . \mathrm{s} / \mathrm{m}$. It is adjusted by a trial-and-error process.

Table 1 Parameters for simulation of the proposed manipulator.

\begin{tabular}{ccc}
\hline Parameter Value & Unit \\
\hline$L$ & 200 & $(\mathrm{~mm})$ \\
$K$ & 0.25 & $(\mathrm{~N} / \mathrm{mm})$ \\
$l_{0 b}, \delta_{e b}$ & 140 & $(\mathrm{~mm})$ \\
$l_{0 c}, \delta_{e c}$ & 100 & $(\mathrm{~mm})$ \\
$l_{p}$ & 20 & $(\mathrm{~mm})$ \\
\hline
\end{tabular}

\begin{tabular}{ccc}
\hline Parameter & Value & Unit \\
\hline$O_{p}$ & {$[0500]$} & $(\mathrm{mm})$ \\
$m_{b}$ & 0.25 & $(\mathrm{~kg})$ \\
$m_{p}$ & 0.25 & $(\mathrm{~kg})$ \\
{$\left[\rho_{\text {bmin }}, \rho_{\text {bmax }}\right]$} & {$[200,500]$} & $(\mathrm{mm})$ \\
{$\left[\rho_{\text {cmin }}, \rho_{\text {cmax }}\right]$} & {$[20,170]$} & $(\mathrm{mm})$ \\
\hline
\end{tabular}



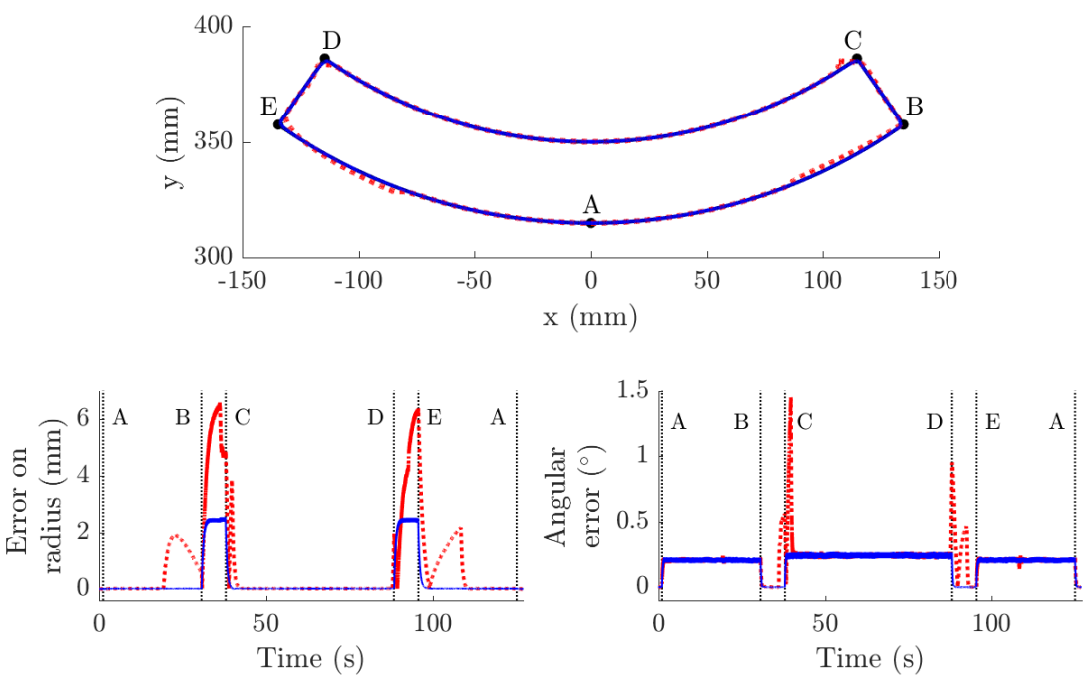

Fig. 3 Top: Cartesian trajectories without (dotted red line) and with (solid blue line) optimization. Bottom: Errors on the RCM constraint. In dotted red and in solid blue the curves obtained using the control schemes proposed in (13) and in (14) respectively.

A comparison with $(k \neq 0)$ and without $(k=0)$ optimization is performed. The end-effector trajectory is displayed in Fig. 3. During the whole process, the manipulator respects the RCM constraint. End-effector path is controlled by modifying the radius value $R_{0}$ (Fig. 1). The manipulator end-effector follows the sequence of points $\mathrm{ABCDEA}$. Along $\mathrm{CD}, R_{0}=200 \mathrm{~mm}$ and along $\mathrm{BE}, R_{0}=235 \mathrm{~mm}$. The angle $\varphi$ varies between -35 and $35^{\circ}$. The evaluation is carried out with an end-effector velocity of $5 \mathrm{~mm} / \mathrm{s}$ and $\mathbf{P}=-\operatorname{diag}(2,2,0.1)$. For the cost function minimization we choose $p_{1}=p_{2}=1$ and $k=10^{6}$. The latter is chosen through a trial-and-error method.

The obtained Cartesian trajectories and errors on the radius and the angle are displayed in Fig 3. The end-effector follows the desired trajectory with average path tracking errors of 0.88 (resp. 0.28) $\mathrm{mm}$ on the radius and 0.22 (resp. 0.19) ${ }^{\circ}$ on the angle without (resp. with) optimization. In Fig. 3, a distortion of the Cartesian trajectory can be observed when the simple pseudo-inverse is used. It is due to the saturation of the joint positions. More specifically, it is caused by the actuators of the bar-actuated SC which reach their minimal bounds and the ones of the cable-actuated SC which reach their maximal bounds. When the control scheme with optimization is used some errors caused by the dynamics can still be observed. However, joint position saturation and tension limits are avoided. 


\section{Conclusions and perspectives}

In this paper, the kinematic model of a tensegrity-based manipulator for a medical application was derived. It was then used to develop and achieve early-stage tests of Jacobian-based control schemes. Optimization of the joint positions was proposed to address actuator saturation and tension issues taking advantage of the tensegrity mechanism redundancy. Promising results are obtained even though only local optimization is considered. Implementation of global optimization is a first perspective of this work. The use of redundancy to minimize other criteria such as the potential energy to improve energy consumption and safety will also be considered.

Acknowledgements This work is supported by the Investissements d'Avenir program (Robotex ANR-10-EQPX-44, Labex CAMI ANR-11- LABX-0004), the French National Research Agency (ANR Multiflag, ANR-16-CE33-0019) and by the Grand Prix Scientifique 2018, Fondation Charles Defforey, Institut de France.

\section{References}

1. Aldrich, J.B., Skelton, R.E.: Time-energy optimal control of hyper-actuated mechanical systems with geometric path constraints. In: Proceedings of the 44th IEEE Conference on Decision and Control, pp. 8246-8253 (2005)

2. Arsenault, M., Gosselin, C.M.: Kinematic and static analysis of a planar modular 2-DoF tensegrity mechanism. In: Proceedings 2006 IEEE International Conference on Robotics and Automation, 2006. ICRA 2006., pp. 4193-4198 (2006)

3. Bayat, J., Crane Carl D., I.: Closed-Form Equilibrium Analysis of Planar Tensegrity Structures. ASME. International Design Engineering Technical Conferences and Computers and Information in Engineering Conference 8(31), 13-23 (2007)

4. Begey, J., Vedrines, M., Andreff, N., Renaud, P.: Toward a design method for tensegrity-based medical robots. In: Surgetica: Computer-Assisted Medical Interventions. Scientific problems, tools and clinical applications. (2019)

5. Boehler, Q., Charpentier, I., Vedrines, M., Renaud, P.: Definition and Computation of Tensegrity Mechanism Workspace. J. Mechanisms Robotics 7(4), 044,502-044,502-4 (2015)

6. Juan, S.H., Mirats Tur, J.M.: Tensegrity frameworks: Static analysis review. Mechanism and Machine Theory 43(7), 859-881 (2008)

7. L Bakker, D., Matsuura, D., Takeda, Y., Herder, J.: Design of an Environmentally Interactive Continuum Manipulator. In: The 14th IFToMM World Congress (2015)

8. Liégeois, A.: Automatic Supervisory Control of the Configuration and Behavior of Multibody Mechanisms. IEEE Transactions on Systems, Man, and Cybernetics 7(12), 868-871 (1977)

9. Siciliano, B.: Kinematic control of redundant robot manipulators: A tutorial. J Intell Robot Syst 3(3), 201-212 (1990)

10. Snelson, K.: Continuous tension, discontinuous compression structures (1965)

11. Sultan, C.: Tensegrity deployment using infinitesimal mechanisms. International Journal of Solids and Structures 51(21), 3653-3668 (2014)

12. Sumi, S., Böhm, V., Schale, F., Zimmermann, K.: Compliant Gripper Based on a Multistable Tensegrity Structure. In: New Trends in Mechanism and Machine Science, Mechanisms and Machine Science, pp. 143-151. Springer, Cham (2017)

13. Wenger, P., Chablat, D.: Kinetostatic Analysis and Solution Classification of a Planar Tensegrity Mechanism. In: S. Zeghloul, L. Romdhane, M.A. Laribi (eds.) Computational Kinematics, Mechanisms and Machine Science, pp. 422-431. Springer International Publishing (2018) 